Revista Mexicana de Astronomía y Astrofísica, 56, 29-38 (2020)

(c) 2020: Instituto de Astronomía, Universidad Nacional Autónoma de México

https://doi.org/10.22201/ia.01851101p.2020.56.01.05

\title{
THE JET/COUNTERJET SYMMETRY OF THE HH 212 OUTFLOW
}

\author{
A. Noriega-Crespo ${ }^{1}$, A. C. Raga ${ }^{2}$, V. Lora ${ }^{3}$, and J. C. Rodríguez-Ramírez ${ }^{4}$ \\ Received February 22 2019; accepted November 212019
}

\begin{abstract}
We present Spitzer (IRAC) images and a VLT $2.1 \mu \mathrm{m}$ image of the HH 212 outflow. We find that this outflow has a strong symmetry, with jet/counterjet knot pairs with $\Delta x<1^{\prime \prime}$ position offsets. We deduce that the jet/counterjet knots are ejected with time differences $\Delta \tau_{0} \approx 6 \mathrm{yr}$ and velocity differences $\Delta v_{0} \approx 2 \mathrm{~km} \mathrm{~s}^{-1}$. We also analyze the deviations of the knot positions perpendicular to the outflow axis, and interpret them in terms of a binary orbital motion of the outflow source. Through this model, we deduce a $\approx 0.7 M_{\odot}$ mass for the outflow source, and a separation of $\approx 80 \mathrm{AU}$ between the components of the binary (assuming equal masses for the two components). Finally, using the IRAC data and the VLT $2.1 \mu \mathrm{m}$ image we measure the proper motion velocities, obtaining values from 50 to $170 \mathrm{~km} \mathrm{~s}^{-1}$.
\end{abstract}

\section{RESUMEN}

Presentamos imágenes del Spitzer (IRAC) y una imagen del VLT a $2.1 \mu \mathrm{m}$ del flujo HH 212. Encontramos que este flujo tiene una fuerte simetría, con pares de nudos en el chorro/contrachorro con diferencias de posición $\Delta x<1^{\prime \prime}$. Deducimos que los pares de nudos chorro/contrachorro son expulsados con diferencias de tiempo $\Delta \tau_{0} \approx 6$ yr y de velocidad $\Delta v_{0} \approx 2 \mathrm{~km} \mathrm{~s}^{-1}$. También analizamos las desviaciones de las posiciones de los nudos perpendiculares al eje del flujo, y las interpretamos como resultado de un movimiento binario orbital de la fuente. A través de este modelo, deducimos una masa de $\approx 0.7 M_{\odot}$ para la fuente, y una separación de $\approx 80 \mathrm{AU}$ para la binaria (suponiendo masas iguales para sus dos componentes). Finalmente, usamos los datos de IRAC y la imagen del VLT a $2.1 \mu \mathrm{m}$ para medir las magnitudes de los movimientos propios, obteniendo valores de 50 a $170 \mathrm{~km} \mathrm{~s}^{-1}$.
Key Words: Herbig-Haro objects - ISM: individual objects: HH212 — ISM: jets and outflows - shock waves — stars: formation — stars: winds, out- flows

\section{INTRODUCTION}

The existence of symmetric emitting knots (at similar distances from the outflow source) along some bipolar Herbig-Haro (HH) outflow systems implies highly synchronized jet/counterjet ejections, and therefore a small spatial extent for the jet production region. This was pointed out by Raga et al. (2011a) in their study of Spitzer Infrared Array Camera (IRAC) images of the HH 34 outflow. In a second paper, Raga et al. (2011b) developed a ballistic jet model which constrained the ejection asymmetries

\footnotetext{
${ }^{1}$ Space Telescope Science Institute, USA.

${ }^{2}$ Instituto de Ciencias Nucleares, UNAM, México.

${ }^{3}$ Instituto de Radioastronomía y Astrofísica Teórica, UNAM, México.

${ }^{4}$ IAG, USP, Brasil.
}

using the observed jet/counterjet structures, and applied the model to the HH 34 and HH 111 outflows.

These analyses of jet/counterjet asymmetries have been carried out with IR Spitzer images in the 4 IRAC channels (centered at 3.6, 4.5, 5.8 and $8.0 \mu \mathrm{m})$. This is because at optical wavelengths, larger jet/counterjet asymmetries are found in the knots close to the outflow source, often with one of the two lobes being undetected because of a large optical extinction (this is the case, e.g., of the HH 34, HH 111 and HH 1/2 outflows). The intrinsic symmetry of the two lobes is then only visible at infrared (IR) wavelengths, as first shown in a quite dramatic way by the $\mathrm{H}_{2} 2.1 \mu \mathrm{m}$ observations of $\mathrm{HH} 111$ of Gredel \& Reipurth (1994).

A clear candidate for this kind of study is the HH 212 outflow, which is an impressive "IR jet", dis- 


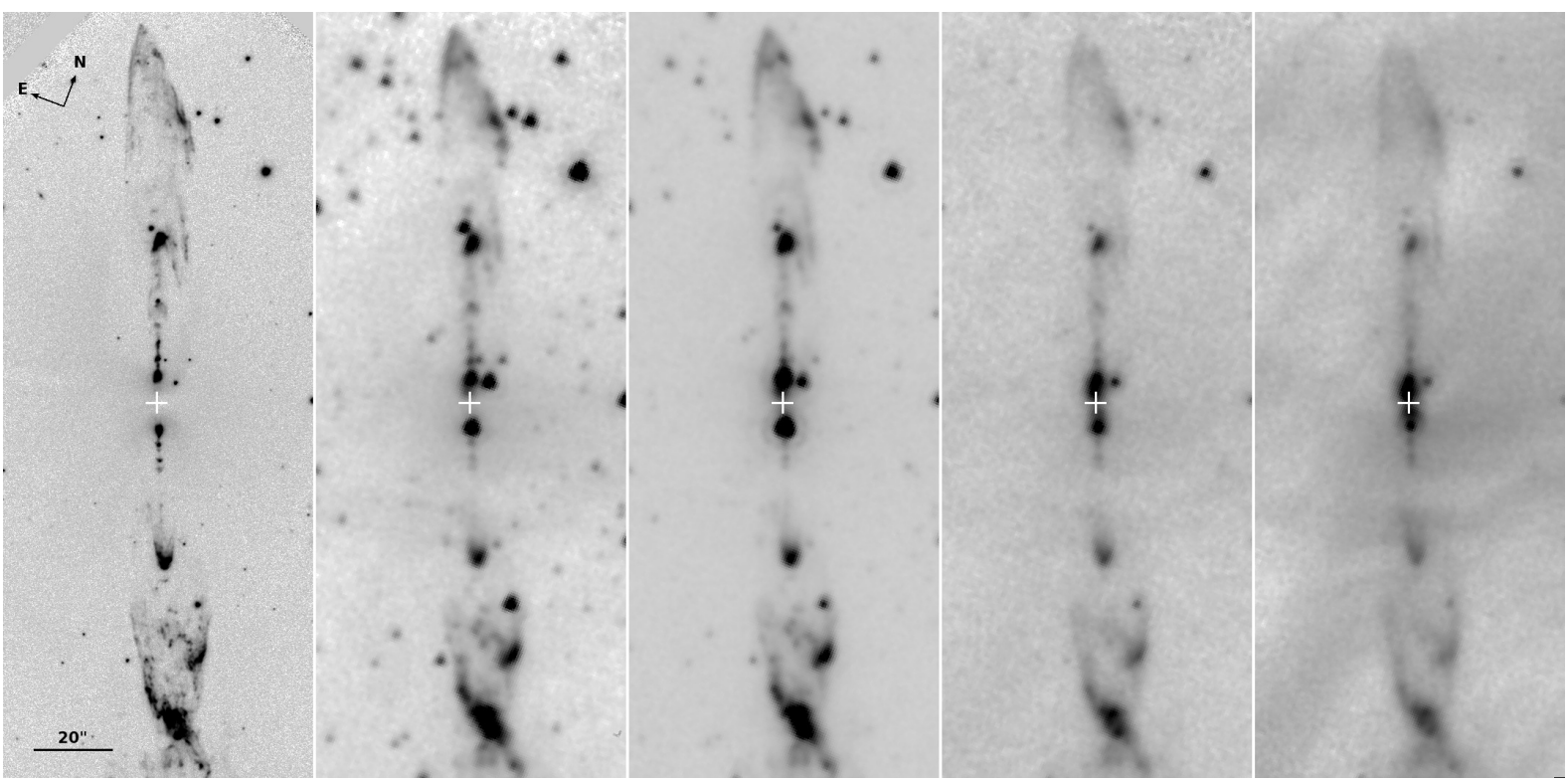

Fig. 1. The $\mathrm{HH} 212$ outflow in $\mathrm{H}_{2} 2.1 \mu \mathrm{m}$ (left frame) and in the four IRAC channels I1-I4, at 3.6, 4.5, 5.8 and 8.0 $\mu \mathrm{m}$ (four frames towards the right). The orientation and the scale of the images are shown in the left frame. The white cross (in all frames) indicates the position of the outflow source.

covered at IR wavelengths by Zinnecker et al. (1998) and with only very faint optical emission (Reipurth et al. 2019). This outflow lies very close to the plane of the sky (Claussen et al. 1998) and is at a distance of approximately 400 pc (Anthony-Twarog 1982, Kounkel et al. 2017). Recent proper motion determinations (Reipurth et al. 2019) show that the jet and the counterjet have a velocity of $\approx 170 \mathrm{~km} \mathrm{~s}^{-1}$.

$\mathrm{H}_{2} 2.1 \mu \mathrm{m}$ observations of $\mathrm{HH} 212$ (Davis et al. 2000, Smith et al. 2007, Correia et al. 2009) show that this outflow has emitting structures with evident jet/counterjet symmetries. We present Spitzer images in the four IRAC channels (I1-I4, at 3.6, 4.5, 5.8 and $8.0 \mu \mathrm{m}$ ) and an archival VLT $2.1 \mu \mathrm{m}$ image of $\mathrm{HH} 212$, and determine the positions of knots along the jet and the counterjet.

We then use the knots within $40^{\prime \prime}$ from the outflow source to calculate jet/counterjet knot position offsets. These offsets (as a function of distance from the outflow source) are then interpreted in terms of the ballistic outflow model of Raga et al. (2011b) in order to constrain the jet/counterjet asymmetries of the ejection process. We also study the deviations of the knot positions perpendicular to the outflow axis, and interpret them in terms of the "orbiting outflow source" model of Masciadri \& Raga (2002).

The paper is organized as follows. The observations are discussed in $\S 2$. The measurement of knot intensities and positions (as well as the determinations of jet/counterjet knot offsets) are presented in $\S 3$. $\S 4$ presents interpretations of the HH 212 measurements, including an application of the jet/counterjet asymmetry model of Raga et al. (2011b), an application of the "orbiting source jet" model of Masciadri \& Raga (2002), an evaluation of the difference in extinction towards the jet and the counterjet, and a discussion of the features of the outflow that do not show a clear jet/counterjet symmetry. The results are summarized in $\S 5$.

\section{THE OBSERVATIONS}

The IRAC data were obtained during the CryoSpitzer mission, program PID 3315 (PI NoriegaCrespo) on "Emission from H2, PAHs and Warm Dust in Protostellar Jets". The data were collected in the four IRAC bands using a 30 sec high dynamic range (HDR) frame time and a 12 point medium scale Reuleaux dither pattern. A small $2 \times 1$ mosaic with a 260 arcsec stepsize was used to capture the outflow within the field of view $(\mathrm{FoV})$ of the four $\operatorname{IRAC}(3.6,4.5,5.8$ and $8.0 \mu \mathrm{m})$ channels. The resulting images have a $0.6^{\prime \prime}$ pixel size.

We used the final reprocessing from the Spitzer Archive with a standard angular resolution of $\mathrm{FWHM} \approx 2$ arcsec. Figure 1 shows the entire outflow in the four bands. As expected, the HH 212 jet itself is brighter at $4.5 \mu \mathrm{m}$, given that some of 
the bright pure (0-0) rotational lines fall within the IRAC Channel 2 bandpass, i.e. S(9) 4.6947, S(10) 4.4096 and $\mathrm{S}(11) 4.1810 \mu \mathrm{m}$ (Noriega-Crespo et al 2004a, 2004b; Looney et al. 2007; Tobin et al. 2007; Ybarra \& Lada 2009; Maret et al. 2009, Raga et al. 2011a; Noriega-Crespo \& Raga 2012). The IRAC Channel 2 map could also be brighter because of the $\mathrm{CO}$ rovibrational lines that fall in its range. However these lines require high temperatures and densities that do not normally occur in protostellar jets. Nevertheless, the jet is detected in all four bands (Figure 1).

We also used a VLT $\mathrm{H}_{2} 2.1 \mu \mathrm{m}$ image obtained with the High Acuity Wide field K-Band Imager (HAWK-I), as part of its Science Verification program (PI Schneider, "How symmetric is a symmetric flow. A deep H2 image of the Herbig Haro object 212") observed in Janauary 2018, and enhanced by the ground-layer adaptive optics module (GRAAL) with an image quality of the order $0.2^{\prime \prime}$. The raw and reduced data are available through the ESO archive. The uncalibrated image has a $0.106^{\prime \prime}$ pixel size (Leibundgut et al. 2018). This image is shown in the left frames of Figures 1 and 2.

\section{THE KNOT POSITIONS AND INTENSITIES}

Figure 2 shows the $\mathrm{H}_{2} 2.1 \mu \mathrm{m}$ and the I1-I4 IRAC maps (with 3.6, 4.5, 5.8 and $8.0 \mu \mathrm{m}$ central wavelengths, respectively) of the HH 212 outflow. The images have been rotated $22.5^{\circ}$ clockwise, so that the outflow axis is parallel to the ordinate. The position of the outflow source (for which we have used the position given in $\S 3.1$ of Galván-Madrid et al. 2004) is shown with a black circle in the central region of the I4 map. On the $\mathrm{H}_{2} 2,1 \mu \mathrm{m}$ map we show the identifications given by Lee et al. (2007) for the $\mathrm{H}_{2}$ knots.

In order to find the positions of the jet/counterjet knots, we convolved the I1-I4 maps with a "Mexican hat" wavelet with a central peak of $\sigma=2$ pixel radius, which has the effect of isolating well defined emission peaks from the spatially more extended emission. On these convolved maps we searched for peaks along the jet axis with an intensity larger than a cutoff value $I_{c}$ (for which we have chosen values of $0.03,0.05,0.1$ and $0.1 \mathrm{mJy} / \mathrm{sterad}$ for the I1, I2, I3 and I4 channels, respectively), and carried out paraboloidal fits in $3 \times 3$ pixel regions (around each of the peaks) to determine the knot positions. This procedure is described in detail by Raga et al. (2017).

The $\mathrm{H}_{2} 2.1 \mu \mathrm{m}$ jet/counterjet knot positions were found on a convolution of this image with a central peak of $\sigma=5$ pixel radius. We selected peaks with at least $10^{-2}$ times the peak knot intensity (which was found for one of the SB knots of Lee et al. 2007) in the convolved frame.

The resulting knot positions are shown as black crosses on the $\mathrm{H}_{2} 2.1 \mu \mathrm{m}$ and I1-I4 maps of Figure 2. It is clear that many of the knots along the NE jet (top half of the frames) have corresponding emitting structures in the SW counterjet (bottom half of the frames).

The knot located $\approx 26^{\prime \prime}$ to the $\mathrm{N}$ of the source (labeled NK7 by Lee et al. 2007) has no detectable counterpart in the counterjet in the IRAC I1-I4 maps. However, in the $\mathrm{H}_{2} 2.1 \mu \mathrm{m}$ image we do detect a faint counterpart along the counterjet (labeled SK7).

In Figure 3 we show the peak intensities of the jet and counterjet knots in the I1-I4 IRAC maps (measured on the convolutions with a $\sigma=2$ pixel radius wavelet) as a function of distance $x$ from the outflow source. This distance is measured along the ordinate of Figures 1 and 2, but (as the offsets of the knots along the abscissa are very small). Almost identical values are obtained if one takes the radial source/knot distances.

The jet and counterjet knots at similar distances from the source have intensities that differ by factors of $\approx 2$. We see that at similar distances from the source:

- in most cases the NE jet knots (in blue) are brighter than the SW counterjet knots (in red),

- the ratios between the jet and counterjet knot intensities generally become smaller for the longer wavelength IRAC channels (see Figure 2).

These trends can be interpreted as the result of a different extinction towards the two outflow lobes, as described in $\S 4.3$.

In the top frame of Figure 4 we show the peak $\mathrm{H}_{2} 2.1 \mu \mathrm{m}$ intensities of the jet and counterjet knots (measured on the convolution with a $\sigma=5$ pixel radius wavelet) as a function of distance $x$ from the outflow source. The intensities are given in units of the peak intensity of the SK1 knot (see Figure 2). The jet and counterjet knots at similar distances from the source have intensities that differ by factors of $\approx 3$, except for knots NK7 and SK7 (at $\approx 26^{\prime \prime}$ from the source) which have intensities that differ by a factor of $\approx 10$.

We now use the $\mathrm{H}_{2} 2.1 \mu \mathrm{m}$ map (which has a higher angular resolution than the IRAC maps) to define jet/counterjet knot associations with pairs of knots which have values of $|x|$ (the distance to the 


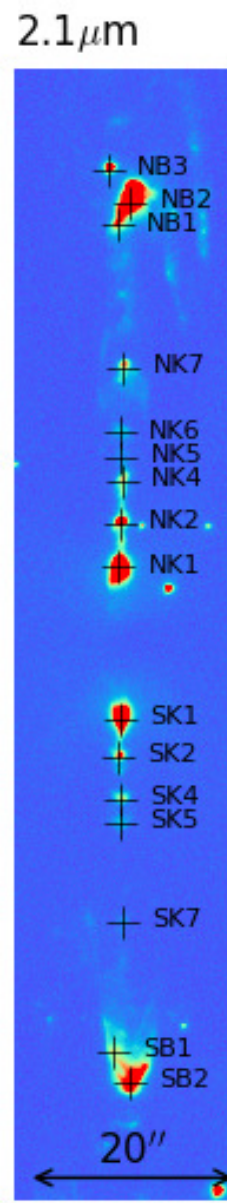

I1

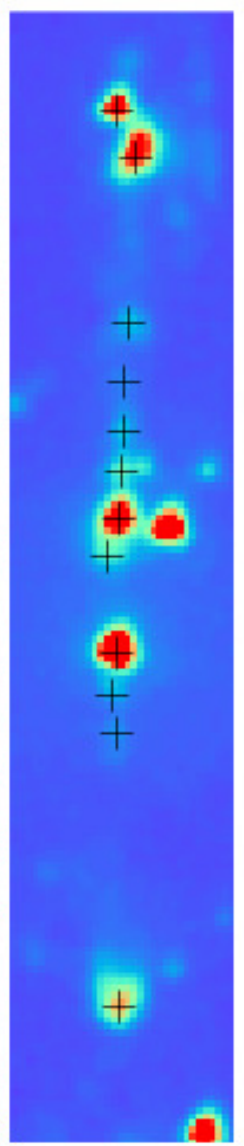

12

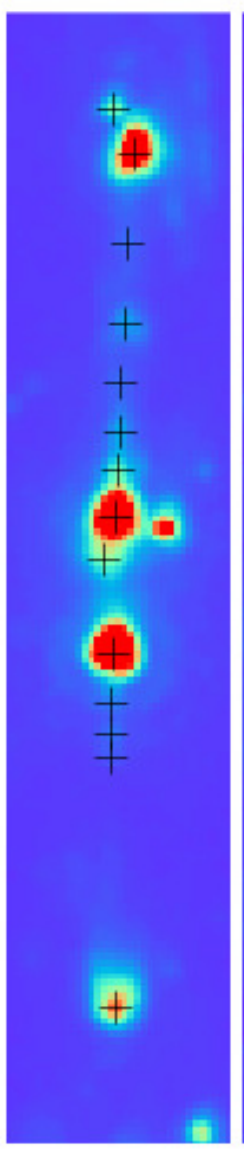

13

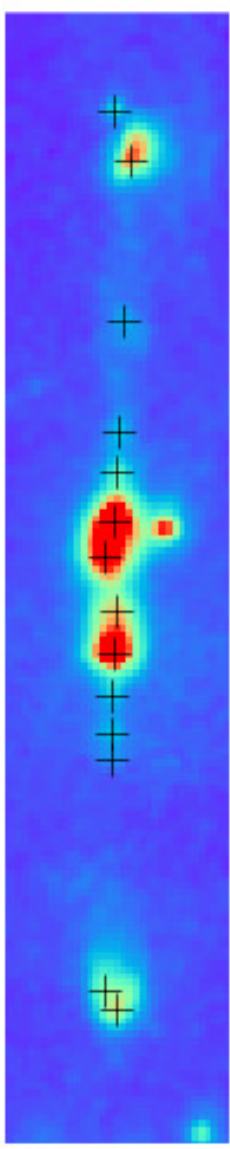

14

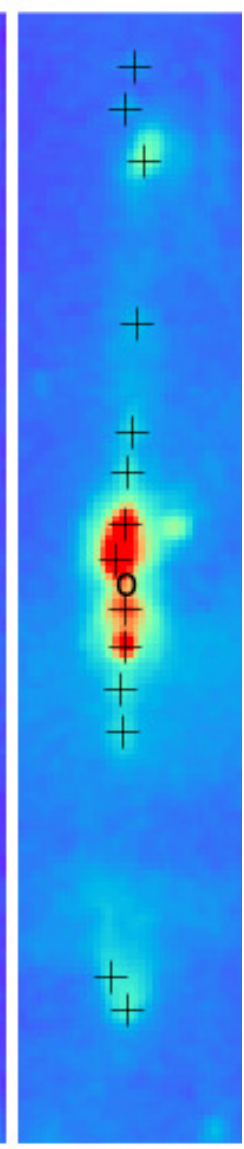

Fig. 2. The central region of the $\mathrm{HH} 212$ outflow in in $\mathrm{H}_{2} 2.1 \mu \mathrm{m}$ (left frame) and in the four IRAC channels (I1-I4, with 3.6, 4.5, 5.8 and $8.0 \mu \mathrm{m}$ central wavelengths, respectively). The ordinate points to the NNE (at $22.5^{\circ}$ clockwise from N). The circle in the central region of the I4 map is the position of the source. The crosses indicate the positions of knots along the jet/counterjet (see the text). The knot identifications of Lee et al. (2007) are given on the $\mathrm{H}_{2} 2.1 \mu \mathrm{m}$ map. The images are displayed with a linear color scale. The color figure can be viewed online.

outflow source) differing by less than $2^{\prime \prime}$. For these pairs of knots, we calculate the jet-counterjet knot position offsets $\Delta x=x_{j}-x_{c j}$ as a function of $x=x_{j}$. In the bottom frame of Figure 4 we show the resulting $\Delta x$ (crosses) and $(\Delta x)^{2}$ (squares) as a function of $x$. This plot shows a trend of marginally increasing jet-counterjet knot position offsets with distance from the outflow source.

In Figure 5 we show the $(x, y)$ knot positions of the inner $\mathrm{H}_{2} 2.1 \mu \mathrm{m}$ knots, with $x$ measured along (positive values for the $\mathrm{N}$ jet) and $y$ across the outflow axis (positive values to the $\mathrm{E}$ ) from the position of the outflow source. It appears that the knots with $|x|<30^{\prime \prime}$ have offsets (with respect to the outflow direction) with a jet/counterjet mirror symmetric pattern. This result is discussed in more detail below.
Finally, we used the new $\mathrm{H}_{2} 2.1 \mu \mathrm{m}$ image (obtained in January 2018) together with the IRAC I1I4 maps (obtained in February 2005) to estimate the proper motions of the HH 212 knots within $50^{\prime \prime}$ from the outflow source. This of course gives only rough estimates of the proper motions, since different knot morphologies in the different spectral bands can in principle lead to position offsets that are not due to proper motions.

We proceeded as follows. For the knots that are present in all the I1-I4 IRAC maps, we first calculate the average positions (along and across the outflow axis), and the standard deviations of these positions. We then use these I1-I4 "first epoch" average knot positions to calculate the knot proper motions together with their corresponding positions in the 


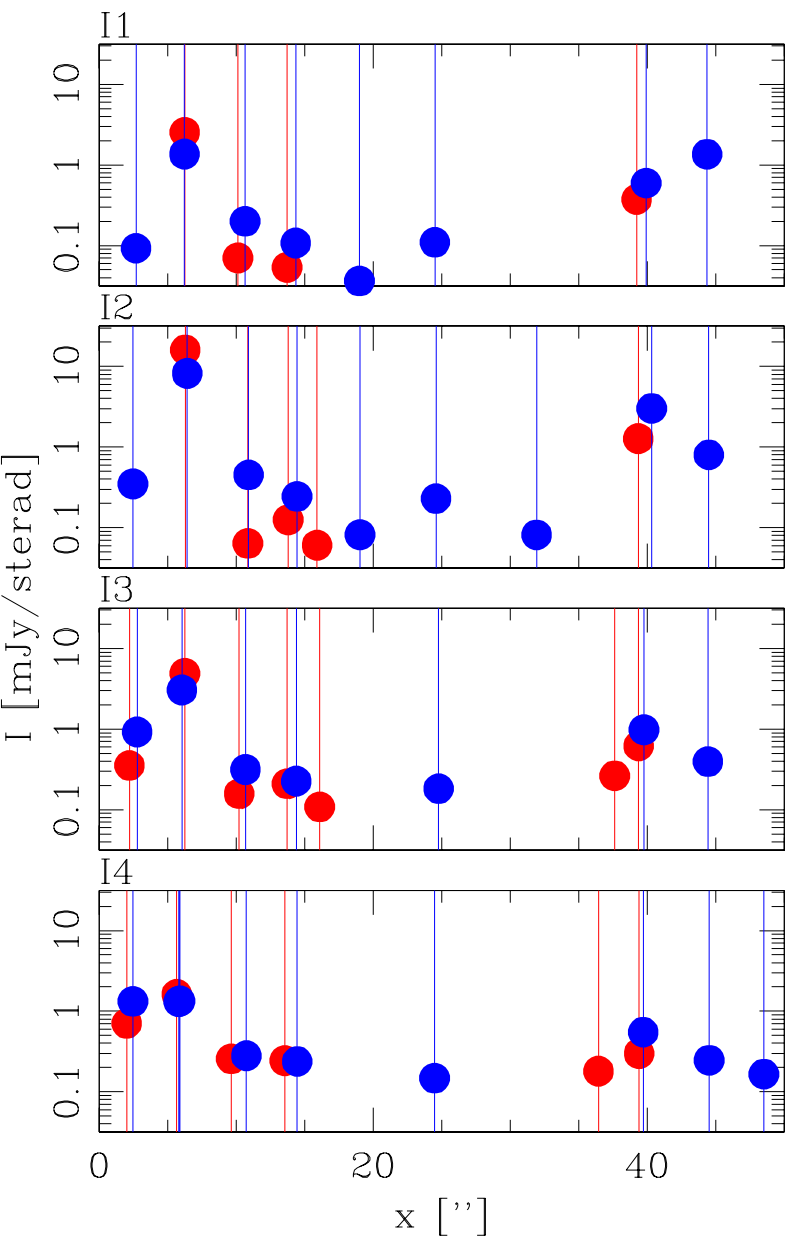

Fig. 3. Fluxes measured for the knots along the jet (blue circles with vertical lines) and counterjet (red circles) as a function of distance $x$ along the outflow axis in the I1-I4 IRAC channel maps. The fluxes are given in $\mathrm{mJy} / \mathrm{sterad}$. The vertical lines are shown so as to highlight the occurrence of jet/counterjet knot pairs with closely matched positions. The color figure can be viewed online.

$\mathrm{H}_{2} 2.1 \mu \mathrm{m}$ "second epoch" map. The proper motion velocities calculated from these knot offsets (assuming a distance of $400 \mathrm{pc}$ to $\mathrm{HH} 212$ ) are given in Table 1.

This table gives the knot identifications (shown in the left frame of Figure 2), the positions $x$ along the outflow axis (measured in the $\mathrm{H}_{2} 2.1 \mu \mathrm{m}$ map, with positive $x$ pointing along the $\mathrm{N}$ jet), and the proper motion velocities along (positive values to the $\mathrm{N}$ ) and across (positive values to the $\mathrm{W}$ ) the outflow axis with their errors (in parentheses). Even though the errors shown are quite small for most of the knots, it is likely that there are larger systematic errors due to the fact that we compare images with different emission features.
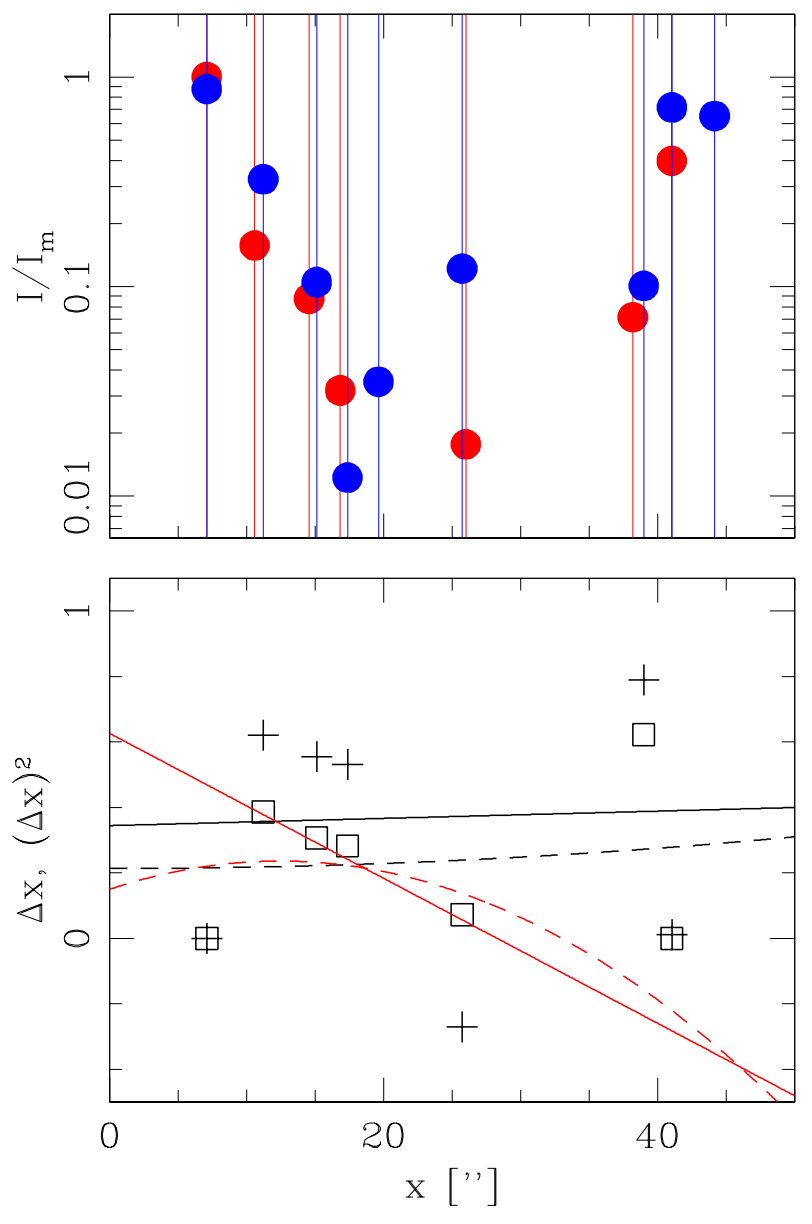

Fig. 4. Top frame: Fluxes measured for the knots along the jet (blue circles with vertical lines) and counterjet (red circles) as a function of distance $x$ along the outflow axis in the $\mathrm{H}_{2} 2.1 \mu \mathrm{m}$ map. The fluxes are given in units of the flux of the SK1 knot. Bottom frame: jet/counterjet knot offsets $\Delta x$ (crosses) and the squares $(\Delta x)^{2}$ of these values (squares) as a function of distance from the source. The results of the linear (black solid lines) and quadratic fits (black dashed lines) to the $\Delta x$ and $(\Delta x)^{2}$ vs. $x$ dependencies are shown (see the text). The red lines are the corresponding fits to the points with $x<30^{\prime \prime}$ only. The jet/counterjet knot offsets have measurements errors of $\approx 0.05^{\prime \prime}$ (corresponding to $\approx 1 / 2$ pixel). The color figure can be viewed online.

The determined proper motion velocities are well aligned with the directions of the jet and the counterjet axes, except for knot NB3. This knot has a motion directed to the NW, which could indicate that it does not belong to the HH 212 outflow or that it has a substantially different morphology in $\mathrm{H}_{2} 2.1 \mu \mathrm{m}$ than in the other spectral bands. 


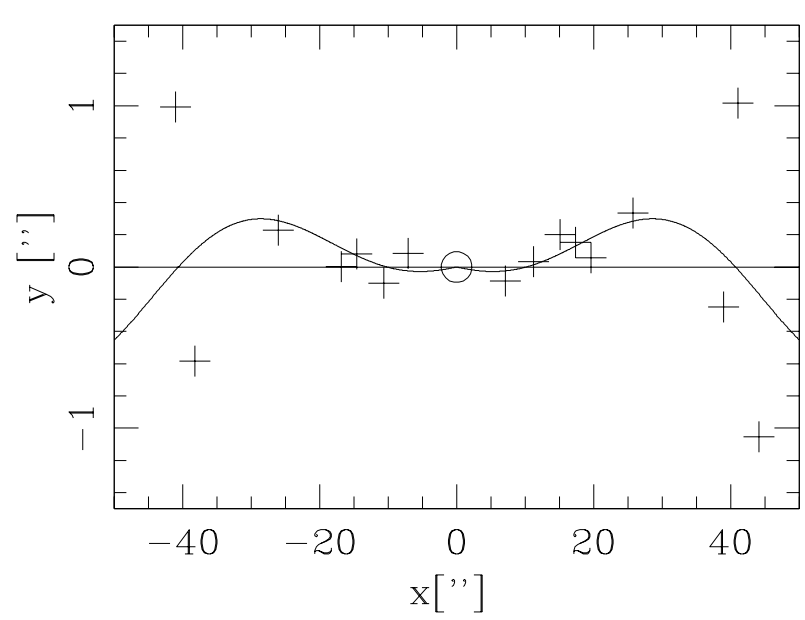

Fig. 5. Positions of the inner HH 212 knots measured on the $\mathrm{H}_{2} 2.1 \mu \mathrm{m}$ frame. The $x$ coordinate lies along (positive $x$ to the $\mathrm{N}$ ), and the $y$ coordinate across the outflow axis (positive $y$ to the $\mathrm{E}$ ). The solid curve is a least squares fit to the knots with $|x|<30^{\prime \prime}$ of the ballistic "orbiting source jet" of Masciadri \& Raga (2002), as described in the text. The positions along and across the outflow axis have errors of $\approx 0.03^{\prime \prime}$.

Most of the knots have axial velocities in the range from $\approx 50$ to $170 \mathrm{~km} \mathrm{~s}^{-1}$, which is roughly consistent with the previously determined proper motions of the $\mathrm{HH} 212$ knots: $115 \pm 50 \mathrm{~km} \mathrm{~s}^{-1}$ by Lee et al. (2015) and somewhat higher velocities by Reipurth et al. (2019). If one compares our results (shown in Table 1) with Table 2 of Reipurth et al. (2019), one finds quite good agreements for the motions of most of the knots present in both tables (note that the knot that we have labeled NB3 does not correspond to the knot with the same denomination in Reipurth et al. 2019).

\section{INTERPRETATION OF THE RESULTS}

\subsection{The Jet/Counterjet Knot Position Asymmetries}

We use the jet/counterjet knot offsets to constrain the jet/counterjet asymmetries along the outflow axis shown in Figure 4. We do this using the model of Raga et al. (2011b). In this model, one assumes that:

- the knots travel ballistically,

- the jet/counterjet knot pairs are ejected with velocities that differ by $\Delta v$ (positive values indicating a faster knot along the jet), with this velocity difference following a uniform distribution with a mean value $v_{0}$ and a half-width $\Delta v_{0}$,
TABLE 1

PROPER MOTIONS OF THE HH 212 KNOTS

\begin{tabular}{crrr}
\hline Knot & \multicolumn{1}{c}{$\begin{array}{c}v_{x} \\
\mathrm{~km} \mathrm{~s}^{-1}\end{array}$} & $\begin{array}{c}v_{y} \\
\mathrm{~km} \mathrm{~s}^{-1}\end{array}$ \\
\hline NK1 & 7.1 & $141(30)$ & $-3(12)$ \\
NK2 & 11.2 & $69(15)$ & $-1(12)$ \\
NK4 & 15.1 & $105(7)$ & $1(10)$ \\
NK7 & 25.7 & $170(17)$ & $-28(7)$ \\
NB2 & 41.1 & $165(36)$ & $10(12)$ \\
NB3 & 44.2 & $-44(10)$ & $-29(17)$ \\
SK1 & -7.1 & $-145(39)$ & $13(21)$ \\
SK2 & -10.6 & $-55(64)$ & $48(22)$ \\
SK4 & -14.6 & $-128(13)$ & $14(10)$ \\
SB2 & -41.0 & $-251(11)$ & $32(12)$ \\
\hline
\end{tabular}

- the knot pairs are ejected with a time-difference $\Delta \tau$ (positive values indicating an earlier jet knot ejection), with the time-difference following a uniform distribution with mean value $\tau_{0}$ and a half-width $\Delta \tau_{0}$.

The values of $v_{0}, \Delta v_{0}, \tau_{0}$ and $\Delta \tau_{0}$ can then be determined by carrying out a linear fit to the $\Delta x$ vs. $x$ trend and a quadratic fit to the $(\Delta x)^{2}$ vs. $x$ trend observed in a particular jet/counterjet system. The mean values and half-widths of the ejection velocity and time distributions can be found from the coefficients of these fits using equations (4) and (6) of Raga et al. (2011b).

We carry out the linear and quadratic fits to the $\Delta x$ vs. $x$ and the $(\Delta x)^{2}$ vs. $x$ values (respectively) obtained from the $\mathrm{H}_{2} 2.1 \mu \mathrm{m}$ map. The results of these fits are shown with solid and dashed lines, respectively, in the bottom frame of Figure 4. With the fitting coefficients we determine the characteristics of the asymmetrical jet/counterjet ejection time and velocitiy distributions (see above and Raga et al. 2011b):

- $v_{0}=(0.16 \pm 0.17) \mathrm{km} \mathrm{s}^{-1}, \Delta v_{0}=(1.60 \pm$ $0.12) \mathrm{km} \mathrm{s}^{-1}$,

- $\tau_{0}=(4.38 \pm 1.54) \mathrm{yr}, \Delta \tau_{0}=(6.77 \pm 4.74) \mathrm{yr}$.

These parameters were derived assuming a distance of $400 \mathrm{pc}$ and a flow velocity of $(170 \pm 30) \mathrm{km} \mathrm{s}^{-1}$ for HH 212 (see Reipurth et al. 2019).

In other words, the jet/counterjet knot position asymmetries of HH 212 can be explained with: 
- an ejection velocity asymmetry with a distribution centered at 0 (i.e., the value of $v_{0}$ determined from the fits is not significantly different from 0 , see above) and half-width of $\approx 1.6 \mathrm{~km} \mathrm{~s}^{-1}$,

- an ejection time asymmetry with a distribution centered at $\approx 4 \mathrm{yr}$, and a (barely significant) width of $\approx 7 \mathrm{yr}$.

These results are qualitatively similar to the ones found for the HH 34 jet/counterjet system by Raga et al. (2011b).

It is fair to say that through this analysis we are basically not detecting a significant asymmetry in the jet/counterjet ejections, and are only estimating upper boundaries (of $\approx 2 \mathrm{~km} \mathrm{~s}^{-1}$ for the velocity and $\approx 4 \mathrm{yr}$ for the ejection time) for possible asymmetries in the ejections.

The large uncertainty in our estimate of the ejection asymmetries is illustrated with the following exercise. One could argue that the local intensity maxima of knots NB1, NB2, SB1 and SB2 actually correspond to local features in larger bow shocks (see Figure 2), and therefore the associations NB1-SB1 and NB2-SB2 used to calculate the offsets at $x \approx 40^{\prime \prime}$ (see the two frames of Figure 4) are not necessarily meaningful. Therefore, we repeat the linear and quadratic fits (to the $\Delta x$ and $(\Delta x)^{2}$ vs. $x$ dependencies) using only the knot offsets obtained for $x<30^{\prime \prime}$.

The results of these fits are shown with the solid (linear fit to $\Delta x$ vs. $x$ ) and dashed (quadratic fit to $(\Delta x)^{2}$ vs. $\left.x\right)$ red lines in the bottom frame of Figure 4 . These fits do not yield physical estimates of the ejection variability, since the formalism of Raga et al. (2011b) gives complex values for the derived parameters for the ejection distributions when using the resulting values of the coefficients of these fits.

Given the lack of a clear correlation of the jet/counterjet knot offsets as a function of distance from the source (evidenced by the fact that the results change in a quite drastic way by removing the knots at $x \approx 40^{\prime \prime}$ ) it is probably fairer to just note that the knot offsets have a mean value $\overline{|\Delta x|}=(0.40 \pm 0.29)^{\prime \prime}$. This corresponds to an average time-difference $\overline{\Delta \tau}=\overline{|\Delta x|} / v_{j}=(4.5 \pm 3.3) \mathrm{yr}$ (for a distance of $400 \mathrm{pc}$ and $v_{j}=170 \mathrm{~km} \mathrm{~s}^{-1}$, see above). This estimate is consistent with the $\Delta \tau_{0}=(6.77 \pm 4.74)$ yr width for the ejection time distribution deduced above using the formalism of Raga et al. (2011b) and the fit to all knot offsets shown in the bottom frame of Figure 4 .

\subsection{The Mirror Symmetric Precession Pattern}

The inner jet/counterjet knot positions of HH 212 show sideways deviations from the outflow axis with an apparent "mirror symmetric pattern". In Figure 5 , we see that the jet/counterjet knots within $30^{\prime \prime}$ from the source show trends of larger values of $y$ (i.e., towards the E) with increasing distances from the source. At $x \approx \pm 40^{\prime \prime}$ we see the NB and SB knots (respectively), which show a larger spread of $y$ values, as a result of the larger size of the NB and SB structures.

The simplest explanation of mirror symmetric patterns in jet/counterjet systems is in terms of an orbital motion of the (binary) outflow source. A ballistic, analytic model of this situation was presented by Masciadri \& Raga (2002), for the case of a circular orbit and by González \& Raga (2004), for elliptical orbits.

Noriega-Crespo et al. (2011) used the "circular orbit model" of Masciadri \& Raga (2002) to fit the "mirror symmetric precession pattern" of the HH 111 jet/counterjet system. From this fit, they derived estimates of the orbital parameters and stellar masses of the assumed binary source of the HH 111 system.

We follow these authors, and carry out a least squares fit of the jet/counterjet locci:

$$
\begin{aligned}
& y=\kappa x \sin \left(\frac{2 \pi}{\tau_{o} v_{j}} x-\psi\right), \\
& z=\kappa x \cos \left(\frac{2 \pi}{\tau_{o} v_{j}} x-\psi\right),
\end{aligned}
$$

where $x$ is the axial coordinate and $(y, z)$ are the axes parallel to the orbital plane (with $y$ being parallel to the plane of the sky). Also, $\psi$ is the orbital phase, $\tau_{o}$ the orbital period, and $\kappa=v_{o} / v_{j}$ the ratio between the orbital and jet velocities. These equations (see Noriega-Crespo et al. 2011) correspond to the small orbital radius limit of the circular orbit model of Masciadri \& Raga (2002). We also assume that the outflow is ejected perpendicular to the orbital plane.

We project equations (1-2) onto the plane of the sky assuming an angle of $5^{\circ}$ between the outflow axis and this plane (see Reipurth et al. 2019), and carry out a least squares fit to the mirror symmetric pattern of the knots within $30^{\prime \prime}$ of the HH 212 source. From this fit, we obtain:

- $\kappa=0.011 \pm 0.001$, corresponding to an orbital velocity $v_{o}=(1.87 \pm 0.17) \mathrm{km} \mathrm{s}^{-1}$ for the $v_{j}=$ $170 \mathrm{~km} \mathrm{~s}^{-1}$ proper motion velocity of Reipurth et al. (2019), 
- $\tau_{o}=(638 \pm 241) \mathrm{yr}$, where we have also assumed a distance of 400 pc to HH 212.

With these values for the orbital velocity and the orbital period we can derive the orbital radius:

$$
r_{1}=\frac{v_{o} \tau_{o}}{2 \pi}=(40 \pm 15) \mathrm{AU},
$$

and a mass

$$
\frac{\alpha^{3}}{(1+\alpha)^{2}} M_{1}=\frac{\tau_{o} v_{o}^{3}}{2 \pi G}=(0.168 \pm 0.068) M_{\odot},
$$

for the primary (jet source) star. In equation (4), $G$ is the gravitational constant $\alpha=M_{2} / M_{1}$ is the mass ratio of the binary. If we have an equal mass binary (with $\alpha=1$ ), we then obtain a mass $M_{1}=$ $(0.67 \pm 0.27) M_{\odot}$ and a binary separation of $2 r_{1}=$ $(80 \pm 30)$ AU.

We should note that Lee et al. (2015) made a fit of a much tighter precession spiral (with a spatial wavelength $\approx 6^{\prime \prime}$, corresponding to $\mathrm{a} \approx 90 \mathrm{yr}$ period) to the observed $\mathrm{CO} / \mathrm{SiO}$ emission of $\mathrm{HH} 212$. This small scale structure of curved jet segments can also be seen in the NK1-NK4 region of the $\mathrm{H}_{2} 2.1 \mu \mathrm{m}$ jet shown in the left panel of Figure 2. Analogously, the larger scale structure (with a period of $\approx 600 \mathrm{yr}$, see above) we are describing here is also seen in the $\mathrm{CO} / \mathrm{SiO}$ map (Figure 8 of Lee et al. 2015).

\subsection{The Jet/Counterjet Extinction}

As discussed in $\S 3$, the jet knots (blue points) in the IRAC channel maps are generally brighter than the counterjet knots (red points, see Figure 3) at the same distances from the outflow source, especially for the shorter wavelengths channels (I1 and I2). In order to quantify this effect, we calculate the $I_{j} / I_{c j}$ jet-to-counterjet knot intensity ratios for the three knots at distances $x=5 \rightarrow 15^{\prime \prime}$ (from the outflow source), and use these ratios to calculate a mean ratio $\left\langle I_{j} / I_{c j}\right\rangle$ for each of the four IRAC channels. Figure 6 shows the resulting mean jet-to-counterjet $\left\langle I_{j} / I_{c j}\right\rangle$ ratios (shown as magnitudes) as a function of $\lambda$ (where $\lambda$ is the central wavelength of the four IRAC channels). In this figure we also show the jetto-counterjet intensity ratio of the knots seen in the $2.1 \mu \mathrm{m}$ map.

We carried out a weighted least squares fits with a linear transformation of the $R=A_{v} / E(B-V)=5$ extinction curve (appropriate for star formation regions) of Fitzpatrick (1999), and we show the results in Figure 6. We show two fits:

- a fit to the jet-to-counterjet intensity ratios measured in the IRAC maps (dashed curve in Figure 6 ). The fit gives a visual extinction to the

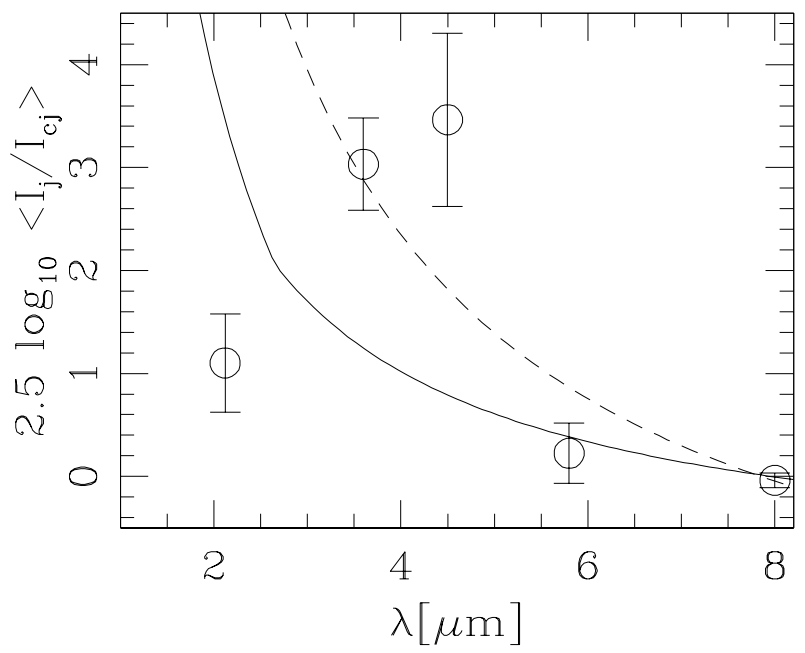

Fig. 6. Mean values of the jet/counterjet intensity ratio (calculated with the three knot pairs at $x=5 \rightarrow 15^{\prime \prime}$, see the text) as a function of central wavelength of the IRAC channels and of the VLT $\mathrm{H}_{2}$ map. The intensity ratios are shown as a magnitude, and as a function of the central wavelength. The solid line shows a least squares fit to the observed line ratios of a linearly transformed $R=5$ extinction curve. The fit to the data gives a higher absorption towards the counterjet of $A_{v}=16.9 \pm 2.3$. The dashed line shows a fit to the intensity ratios with $\lambda>3 \mu \mathrm{m}$.

counterjet larger by $A_{v}=16.9 \pm 2.3$ than the extinction towards the jet,

- a fit to the jet-to-counterjet intensity ratios measured in the IRAC maps and in the $2.1 \mu \mathrm{m}$ map (solid curve in Figure 6). The fit gives a visual extinction to the counterjet larger by $A_{v}=7.2 \pm 1.3$ than the extinction towards the jet.

These of course are estimates only of the difference between the jet and counterjet extinction, and not a determination of the value of the total extinction to the HH 212 outflow.

The measured $I_{j} / I_{c j}$ jet-to-counterjet knot intensity ratios shown in Figure 6 have quite large deviations from the extinction curves. This indicates that the jet and counterjet knots at similar distances from the source have relatively large intrinsic intensity differences, not attributable in a direct way to a difference in the extinction.

\subsection{Knot NKY}

Knot NK7 is located along the NE jet at a distance $x \approx 25^{\prime \prime}$ from the outflow source (see Figures 2 and 3). Lee et al. (2007) show that this knot has a 
very faint $\mathrm{H}_{2} 2.1 \mu \mathrm{m}$ southern counterpart, but they do not detect it in $\mathrm{SiO}$ and $\mathrm{CO}$ (at sub-mm wavelengths). We also see the faint SK7 counterpart to NK7 in our $\mathrm{H}_{2}$ image (see Figure 2). This result, together with the fact that we do not see the southern counterpart of NK7 in the IRAC images, indicates that this knot is intrinsically much brighter along the NE jet than the coresponding ejection along the counterjet, and that this strong brightness asymmetry is not an extinction effect (as the extinction should be much less important at longer wavelengths).

Should we therefore conclude that even though the jet/counterjet ejection from the HH 212 source appears to have a remarkable degree of symmetry (see $\S 4.1$ ), every now and then it produces highly asymmetrical ejections? This is a possible interpretation of the lack of a bright counterpart for the NK7 knot.

Another possible mechanism for producing the observed intensity asymmetry is that knot NK7 corresponds to the merger of two knots (travelling down the jet at slightly different velocities), and that the brightening is associated with the merging process (which produces a knot of boosted shock velocities). If this were the case, we might expect to see a sudden brightening of an "SK7" knot (at $x \approx 25^{\prime \prime}$ from the source) along the counterjet when the corresponding knot merger occurs in the counterjet.

\section{CONCLUSIONS}

We present Spitzer (IRAC) observations and an $\mathrm{H}_{2} 2.1 \mu \mathrm{m}$ image of the $\mathrm{HH} 212$ outflow. In these maps, the general structure of the two outflow lobes is seen (see Figure 1).

For the inner $\approx 1^{\prime}$ of the outflow, we determine the positions of knots along the NE jet and SW counterjet (see Figure 2), and find that they mostly fall into "jet/counterjet knot pairs" (with distances from the source differing by at most $\left.\approx 1.2^{\prime \prime}\right)$. We then calculate the jet/counterjet knot offsets $\Delta x$ as a function of distance $x$ from the outflow source (see Figure 4). We carry out the analysis of knot position offsets with the $2.1 \mu \mathrm{m}$ map, which has higher angular resolution than the IRAC maps.

We interpret the observed jet/counterjet position offsets with the quasi-symmetric ballistic ejection model of Raga et al. (2011b). Through this exercise we determine that the knot pairs are ejected with time-differences $\Delta \tau_{0} \approx 6$ yr and velocity differences $\Delta v_{0} \approx 2 \mathrm{~km} \mathrm{~s}^{-1}$. These results are similar to the ones obtained for HH 34 by Raga et al. (2011b). Clearly, an appropriate ejection model should have this degree of jet/counterjet coordination.
One can in principle use the determined jet/counterjet ejection coordination to estimate a physical size for the jet production region. In the cool, magnetized ejection mechanisms appropriate for low mass young stars, the signal transmission velocity (which could be either the Alfvén or the sound speed) is expected to lie in the $v_{s} \approx 0.1 \rightarrow 10 \mathrm{~km} \mathrm{~s}^{-1}$ range. We would then predict a size of $L=\Delta \tau_{0} v_{s} \approx$ $0.1 \rightarrow 10 \mathrm{AU}$ for the jet production region. The lower limit of this size range is in agreement with the estimation of Lee et al. (2017) of a $\approx 0.1 \mathrm{AU}$ size for the HH 212 outflow collimation region.

We use the knot positions measured on the $\mathrm{H}_{2} \quad 2.1 \mu \mathrm{m}$ image together with the IRAC maps (which were obtained $\approx 13 \mathrm{yr}$ earlier) to determine proper motions of the knots along the jet and the counterjet. We find generally good agreement with the proper motions obtained by Reipurth et al. (2019) with two $\mathrm{H}_{2} 2.1 \mu \mathrm{m}$ epochs covering an $\approx 8 \mathrm{yr}$ time-interval.

We also analyze the deviations of the knot positions perpendicular to the mean axis of the outflow. These deviations show a mirror symmetric jet/counterjet pattern, which can be interpreted in terms of a ballistic outflow from a source in an orbit around a binary companion. We fit the model of an outflow source in a circular orbit of Masciadri \& Raga (2002) to the observed deviations (see Figure 5). From the model fit we deduce an (80 \pm 30$)$ AU binary separation and a $(0.67 \pm 0.27) M_{\odot}$ mass for the outflow source (assuming that the binary companion has the same mass as the outflow source). This estimate for the separation between the binary components coincides with the $\approx 90 \mathrm{AU}$ radius of the disk around the HH 212 source observed by Codella et al. (2014). Our estimated mass is somewhat larger than the $\approx 0.15 M_{\odot}$ mass estimated by Lee et al. (2006, from observations of an infalling envelope) and the $\approx 0.3 M_{\odot}$ mass estimated by Codella et al. (2014, from the rotation of the disk) for the HH 212 outflow source.

The general structure of HH 212 has an important asymmetry in that the NK7 knot (at $\approx 25^{\prime \prime}$ along the NE jet, see Figures 2 and 3) does not have a comparably bright counterpart along the counterjet. This asymmetry is observed at all wavelengths at which the HH 212 outflow has been observed (see Lee et al. 2007), and therefore cannot be accounted for by differential extinction (see $\S 4.3$ and $\S 4.4$ ). We suggest that the asymmetric knot NK7 could be interpreted as a true ejection asymmetry, or as a recent brightening of the knot due to the merger of two "outflow events". If this latter explanation is correct, 
we might expect a future brightening of a counterjet knot at a comparable distance to the outflow source.

Finally, we use the wavelength dependence of the jet/counterjet intensity ratio (measured in the four IRAC channels) to determine the difference in the extinction to the $\mathrm{HH} 212$ jet and counterjet. We conclude that the extinction towards the counterjet is larger (than the one towards the jet) by $A_{v} \approx 10$ magnitudes. This result is similar to the one found by Raga et al. (2019) for the HH 34 outflow. However, we find large deviations between the extinction curve and the jet/counterjet intensity ratios (as a function of wavelength). This indicates that the jet and counterjet knots at similar distance from the outflow source have relatively large intrinsic intensity differences.

AR acknowledges support from the DGAPAUNAM grant IG100218. JCRR acknowledges the Brazilian agency FAPESP grant 2017/12188-5. We thank an anonymous referee for helpful comments, which gave rise to $\S 4.3$.

\section{REFERENCES}

Anthony-Twarog, B. J. 1982, AJ, 87, 1213

Codella, C., Cabrit, S., Gueth, F. et al. 2014, A\&A, 568, L5

Correia, S., Zinnecker, H., Ridgway, S. T., \& McCaughrean, M. J. 2009, A\&A, 505, 673

Davis, C. J. Berndsen, A., Smith, M. D., Chrysostomou, A., \& Hobson, J. 2000, MNRAS, 314, 241

Fitzpatrick, E. L. 1999, PASP, 111, 63

Galván-Madrid, R., Ávila, R., \& Rodríguez, L. F. 2004, RMxAA, 40, 31

González, R. F. \& Raga, A. C. 2004, RMxAA, 40, 61

Gredel, R. \& Reipurth, B. 1994, A\&A, 289, L19

Kounkel, M., Hartmann, L., Loinard, L., et al. 2017, ApJ, 834, A142
Lee, C.-F., Hirano, N., Zhang, Q., et al. 2015, ApJ, 805, 186

Lee, C.-F., Ho, P. T. P., Beuther, H., et al. 2006, ApJ, 639, 292

Lee, C.-F., Ho, P. T. P., Hirano, N., et al. 2007, ApJ, 659,499

Lee, C.-F., Ho, P. T. P., Li, Z.-Y., et al. 2017, NatAs, 1, 0152

Leibundgut, B., Hibon, P., Kuntschner, H., et al. 2018, Msngr, 172, 8

Looney, L. W., Tobin, J. J., \& Kwon, W. 2007, ApJ, 670, 131

Masciadri, E. \& Raga, A. C. 2002, ApJ, 568, 733

Maret, S., Bergin, E. A., Neufeld, D. A., et al. 2009, ApJ, 698,1244

Noriega-Crespo, A., Morris, P., Marleau, F. R., et al. 2004a, ApJS, 154, 352

Noriega-Crespo, A., Moro-Martin, A., Carey, S., et al. 2004b, ApJS, 154, 402

Noriega-Crespo, A., Raga, V., Stapelfeldt, K. R., \& Carey, S. J. 2011, ApJ, 732, L16

Noriega-Crespo, A. \& Raga, A. C. 2012, ApJ, 750, 101

Raga, A. C., Noriega-Crespo, A., Lora, V., Stapelfeldt, K. R., \& Carey, S. J. 2011a, ApJ, 730, L17

Raga, A. C., Noriega-Crespo, A., Rodríguez-Ramírez, J, C., et al. 2011b, RMxAA, 47, 289 . 2012, ApJ, 748, 103

Raga, A. C., Reipurth, B., Esquivel, A., et al. 2017, RMxAA, 53, 485

Raga, A. C., Reipurth, B., \& Noriega-Crespo, A. 2019, $\mathrm{RMxAA}$, in press

Reipurth, B., Davis, C. J., Bally, J., et al. 2019, AJ, submitted

Smith, M. D., O'Connell, B., \& Davis, C. J. 2007, A\&A, 466,565

Tobin, J. J., Looney, L. W., Mundy, L. G., Kwon, W., \& Hamidouche, M. 2007, ApJ, 659, 1404

Ybarra, J. E. \& Lada, E. A. 2009, ApJ, 695, 120

Zinnecker, H., Bastien, P., Arcoragi, J.-P., \& Yorke, H. W. 1992, A\&A, 265, 726

Zinnecker, H., McCaughrean, M. J., \& Rayner, J. T. 1998, Natur, 394, 862

V. Lora: Instituto de Radioastronomía e Astrofísica Teórica, Universidad Nacional Autónoma de México, Ap.372, 58089 Morelia, Michacán, México.

A. Noriega-Crespo: Space Telescope Science Institute, 3700 San Martin Drive, Baltimore, MD 21218, USA (anoriega@stsci.edu).

A. C. Raga: Instituto de Ciencias Nucleares, Universidad Nacional Autónoma de México, Ap. 70-543, 04510 Cd. Mx., México (raga@nucleares.unam.mx).

J. C. Rodríguez Ramírez: Instituto de Astronomia, Geofísica e Ciências Atmosféricas, Universidade de São Paulo, R. do Matão 1226, 05508-090 São Paulo, SP, Brasil. 